Supporting Information

\title{
Fracture Toughness and Surface Energy Density of Kerogen by Molecular Dynamics Simulations in Tensile Failure
}

\author{
Tianhao $\mathrm{Wu}^{a}$ and Abbas Firoozabadi ${ }^{a} b^{*}$ \\ ${ }^{a}$ Reservoir Engineering Research Institute, Palo Alto, California 94301, USA \\ ${ }^{\mathrm{b}}$ Chemical and Biomolecular Engineering Department, Rice University, Houston, Texas 70057, USA \\ *Corresponding author. Email: af@rerinst.org
}




\section{Size-dependency}

To examine the size-dependency in simulations, we have constructed type II-A kerogen matrices with domain sizes containing 27 and 125 macromolecules. The procedure is the same as constructing the matrix with 60 macromolecules. We perform the Monte Carlo simulations using a probe molecule to calculate the porosity. ${ }^{1-3}$ The probe molecule has the diameter of helium (2.9 $\left.\AA\right)$ based on pcff + . All the three matrices have a similar porosity varying from 2.1 to $2.6 \%$ (Fig. S5). The density is $1.11 \mathrm{~g} / \mathrm{cm}^{3}$, which is in line with the literature data. ${ }^{4}$ The strain-driven process is performed for the matrix of different domain sizes (Fig. S6). In the elastic and plastic regions, the three curves almost overlap. There is a minor deviation in the post-failure region. The larger size matrix provides a smoother plot as expected. There is no appreciable size-dependency of the mechanical properties at this scale.

As for the fracture toughness calculation, it is important to confirm the system covers the full fracture process zone. The size of the fracture process zone can be estimated approximately based on Irwin's formulation for plain strain: ${ }^{5-6}$

$$
r_{p}=\frac{1}{6 \pi}\left(\frac{K_{I c}}{\sigma_{c}}\right)^{2}
$$

We use the type II-A kerogen matrix as an example and assign $K_{\mathrm{Ic}}=0.032 \mathrm{MPa} \cdot \mathrm{m}^{1 / 2}$ and $\sigma_{\mathrm{c}}=180 \mathrm{MPa}(\mathrm{see}$ Table 1). The size of the process zone should be around $1.7 \mathrm{~nm}$, which can be covered in the system. It is much larger than in minerals of high stiffness and low toughness.

To further examine the effect of size on the calculation of fracture toughness, we have investigated a larger system $(\sim 12 \mathrm{~nm})$. We constructed a semi-2D type II-C kerogen matrix $(\sim 12 \mathrm{~nm} \times 12 \mathrm{~nm} \times 2.4 \mathrm{~nm})$ to improve computational efficiency. The same procedure of matrix construction and simulation described in the manuscript is applied except the dimensions (see Fig. S7). The tensile strain is applied in the $y$ direction. There is a similar trend in tensile strength and a steeper curve in the rupture region due to the 
simplification as a semi-2D model (see Fig. S8). The toughness is $0.244 \mathrm{~J} / \mathrm{m}^{2}$, which is close to the results from the simulations we have reported in the manuscript $\left(0.279 \mathrm{~J} / \mathrm{m}^{2}\right)$.

\section{Young's modulus, Poisson's ratio, and surface energy density}

In the elastic region, we obtain Young's modulus $E$, based on:

$$
\begin{gathered}
E=E^{\prime}\left(1-v^{2}\right) \\
E^{\prime}=\frac{\sigma}{\varepsilon}
\end{gathered}
$$

where $E^{\prime}$ is the elastic modulus of plane-strain and $v$ is Poisson's ratio. ${ }^{7}$

Poisson's ratio is obtained from: ${ }^{8}$

$$
\frac{v}{1-v}=\frac{\sigma_{x}}{\sigma_{z}}=\frac{\sigma_{y}}{\sigma_{z}}
$$

where $\sigma_{x}, \sigma_{y}$, and $\sigma_{z}$ are the stresses in the $x, y$, and $z$-direction, respectively.

The surface energy density $\gamma_{s}$ of kerogen is calculated based on the potential energy difference between the solid slab and the same number of molecules in the bulk phase of an infinite solid. The surface energy density is given by ${ }^{9}$

$$
\gamma_{s}=\frac{E_{\text {slab }}-E_{\text {bulk }}}{A_{\text {slab }}}
$$

where $E_{\text {slab }}$ is the total energy of the solid slab, $E_{\text {bulk }}$ is the total energy of the same number of molecules in the bulk phase of an infinite solid, and $A_{\text {slab }}$ is the area of the surface considered. Since the kerogen matrix is a porous medium, the $A_{\text {slab }}$ is set as the surface area difference between the slab and the bulk phase. In the isothermal process, only the potential energy difference is considered. In this study, the bulk phase of kerogen is represented by the matrix with periodic boundary conditions in all three directions (see Fig. S12). The potential energy of the kerogen slab is calculated by using the slab with a non-periodic 
boundary condition in the $z$-direction but periodic boundary conditions in the $x$ and $y$-directions. The surface of the slab is perpendicular to the $z$-direction. The long-range interactions in the $z$-direction are neglected. The bulk phase and the kerogen slab are both relaxed at $0 \mathrm{MPa}$ for $100 \mathrm{ps}$.

\section{Tensile failure in illite}

We perform molecular simulations of tensile failure in illite to calculate the critical energy release rate. The composition in our simulations is $\mathrm{K}_{(x+y)}\left[\mathrm{Al}_{(4-y)} \mathrm{Mg}_{y}\right]\left(\mathrm{Si}_{(8-x)} \mathrm{Al}_{x} \mathrm{O}_{20}\right)(\mathrm{OH})_{4}$ where $x=1$ and $y=0.5 .^{10-11}$ The starting configuration is the pyrophyllite unit cell, and the random substitutions of $\mathrm{Al}(\mathrm{III})$ and $\mathrm{Si}(\mathrm{IV})$ are used. The distribution of potassium is also constructed with random locations. The simulations are performed with ClayFF. The tension is performed in the directions that are perpendicular ( $z$-direction) and parallel ( $x$-direction) to the layers, respectively (see Fig. S13). The plane strain is applied such that the dimensions in the plane perpendicular to the direction of tension are kept constant. The stress-strain curves are presented in Fig. S14. To compare with the results in the literature, the crack area is set to the crosssectional area of the plane perpendicular to the tension. ${ }^{10}$

\section{Estimation of critical stress in kerogen matrix with crack}

Critical stress $\sigma_{c}$ of a sample with a crack length of $2 c$ (see Fig. S15) under plane strain can be estimated based on the theories of Griffith and Irwin ${ }^{12-14}$

$$
\sigma_{c}=\sqrt{\frac{E^{\prime} G_{c}}{\pi c}}
$$

To examine Eq. (S6), we construct three matrices of type II-A kerogen that contain cracks ranging from 1 to $3 \mathrm{~nm}$ (see Fig. S16). We place 60 kerogen macromolecules (type II-A) at random positions in a cubic box with a side length of $20.0 \mathrm{~nm}$. To include the initial fracture in the matrix, we set a dummy region with different sizes in the $y$-direction in the middle of the box. The surface of the dummy region is treated as a virtual wall with the LJ-12/6 potential, which has the energy parameter $\varepsilon / k_{\mathrm{B}}=10 \mathrm{~K}$ and the cutoff 
length of $14 \AA$. The remaining procedures of matrix creation are the same as in the section of Methods in the paper. The dummy region is removed after the annealing process. Then, an extra barostat at 1 bar in the $z$-direction is performed for 100 ps to eliminate the effect of the dummy region on the stress. The procedure of the simulations for tensile failure is the same as in the section of Methods. The critical stresses are 166, 128, and $117 \mathrm{MPa}$ for the matrices with the initial crack with lengths of 1,2, and $3 \mathrm{~nm}$, respectively. However, based on Eq. (S6), the predicted critical stresses are 809, 572, and $467 \mathrm{MPa}$, which are much higher than the calculated results. This large difference may be because the theories of Griffith and Irwin are based on the assumption of homogeneous media in which the pores are much smaller than the crack length. However, kerogen is a porous medium where the pore size is in the same order of magnitude as the crack length at this scale in MD simulations. The critical stress may be affected by the pores significantly. To account for porosity $\phi$, we introduce a correction factor $\alpha$ in terms of the ratio of representative pore radius $r$ to half crack length $c$ as:

$$
\begin{gathered}
\alpha=\left(1-\frac{r}{c}\right)^{n} \exp (-k \phi) \\
\sigma_{t}=\alpha \sqrt{\frac{E^{\prime} G_{c}}{\pi c}}
\end{gathered}
$$

where $n$ and $k$ are fitting parameters. According to the pore size distribution of the type II-A kerogen matrix, the porosity is $2.6 \%$, and the representative pore radius is $0.3 \mathrm{~nm}$ (see Figs. S2 and S4). The results are presented in Fig. S16(d), which can match the critical stress. The fitted parameters are $n=0.6632$ and $k=25.3730$. 
(a) Type I-A

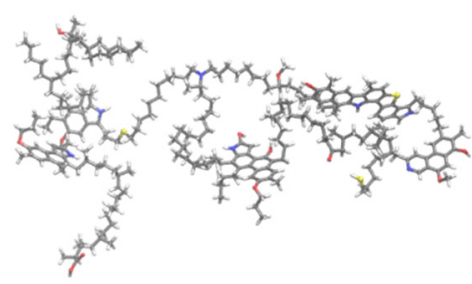

(d) Type II-B

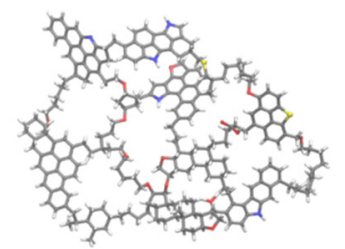

(b) Type II-A

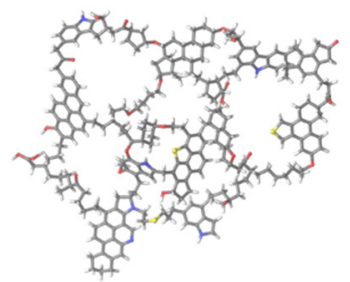

(e) Type II-C

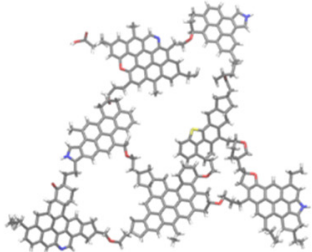

(c) Type III-A

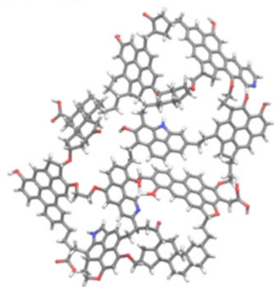

(f) Type II-D

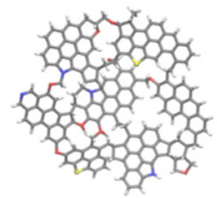

Fig. S1. Molecular structures of kerogen macromolecules. The kerogen macromolecules are constructed based on the work of Ungerer et al. ${ }^{4}$ 
(a) Type I-A
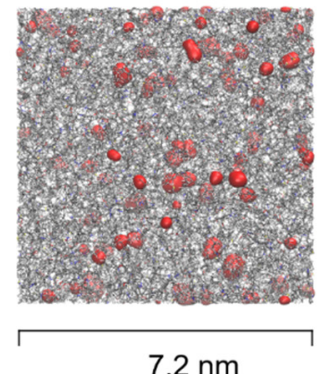

Porosity $=0.6 \%$

(d) Type II-B

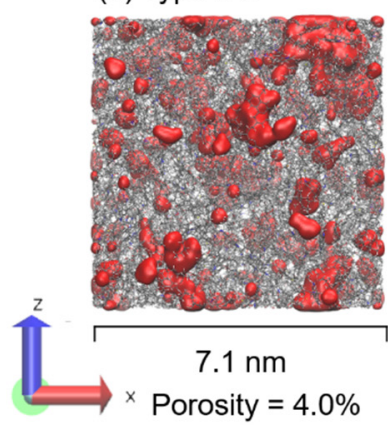

(b) Type II-A

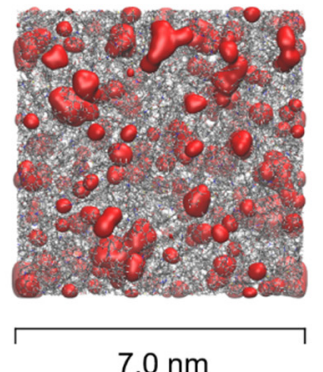

Porosity $=2.6 \%$

(e) Type II-C

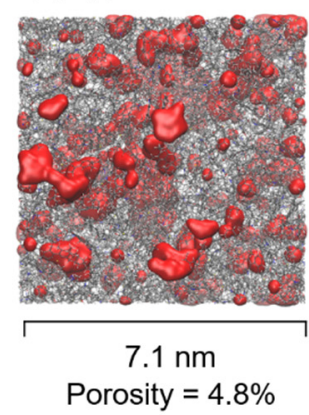

(c) Type III-A

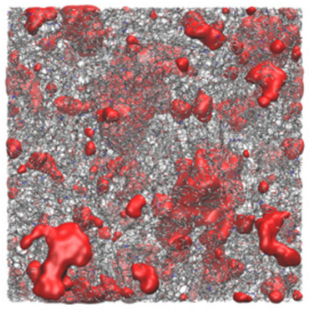

$7.2 \mathrm{~nm}$

Porosity $=7.2 \%$

(f) Type II-D

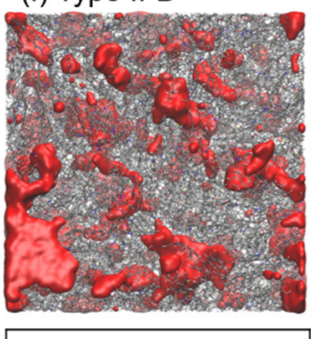

$7.4 \mathrm{~nm}$

Porosity $=9.0 \%$

Fig. S2. Constructed kerogen matrices of different types and maturities. The red color represents the pore space. Probe molecule: helium. 

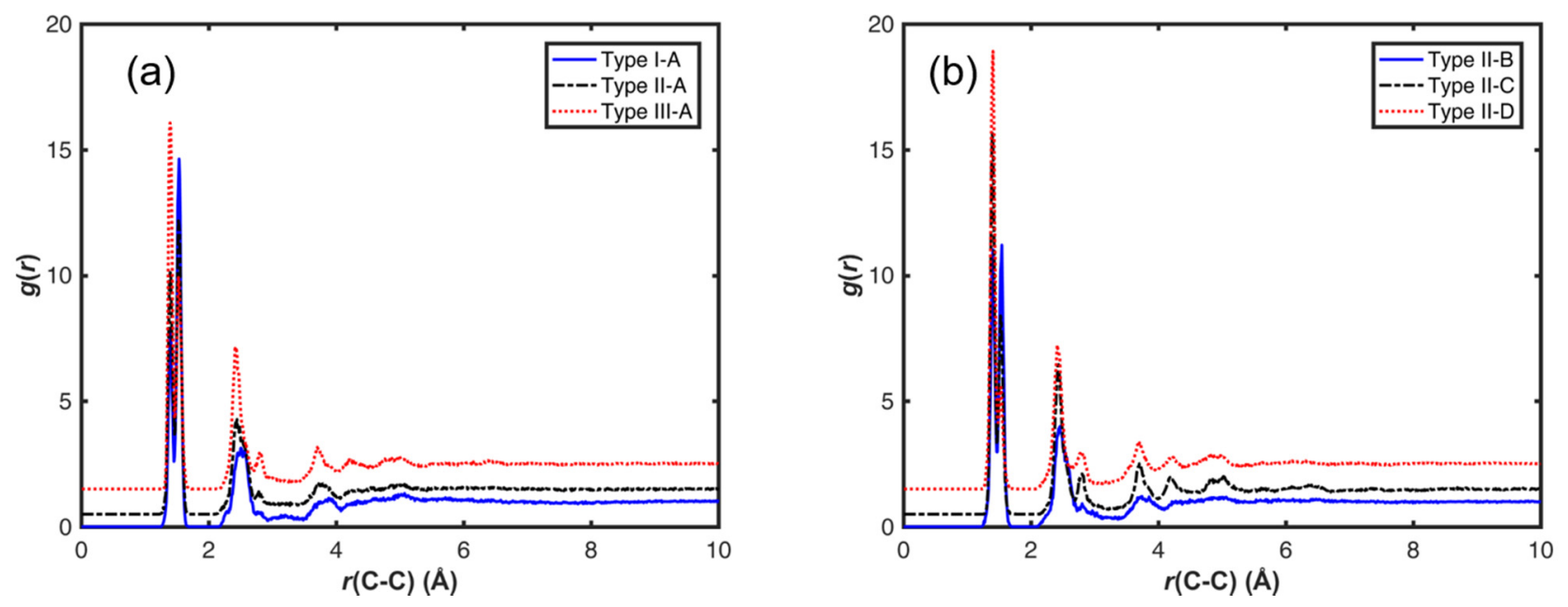

Fig. S3. Radial distribution function between carbon atoms. The simulations are performed at $298 \mathrm{~K}$ and 1 bar. For the sake of clarity, we shifted $g(r)$ with +0.5 for types II-A and II-C, and +1.5 for types III-A and II-D, and with no shift applied for types I-A and II-B. 

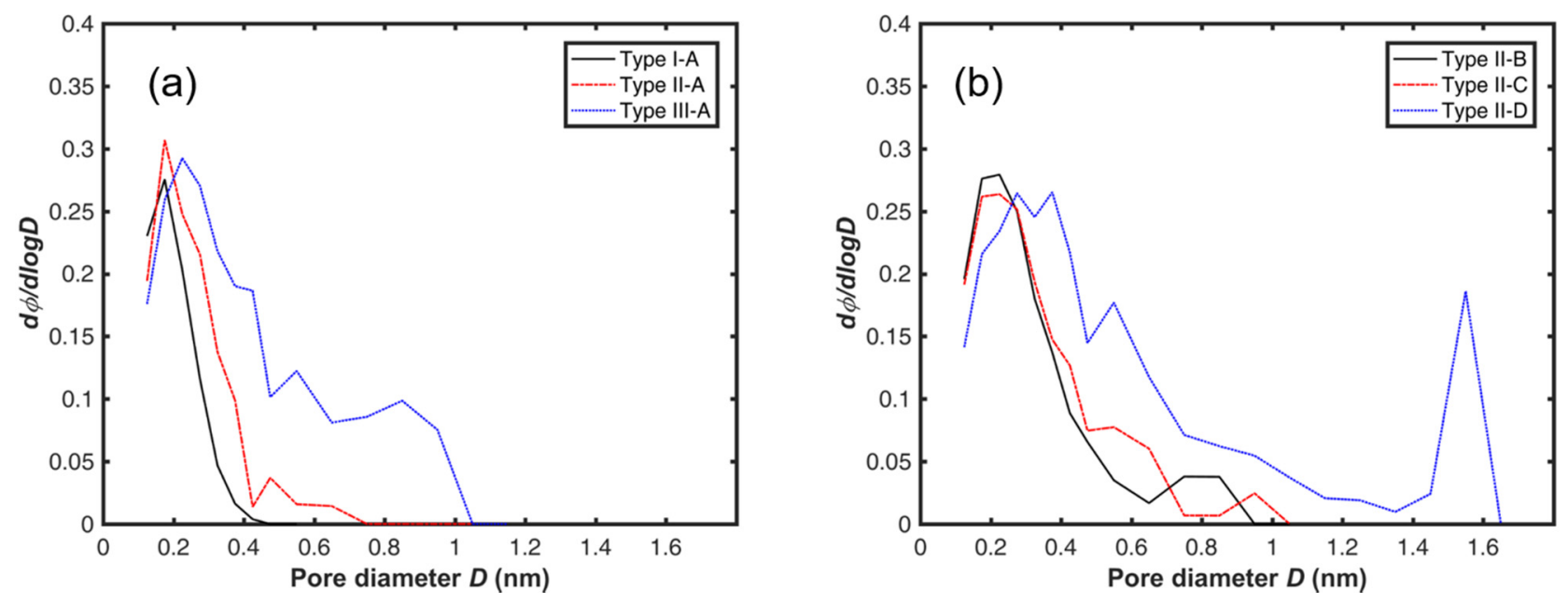

Fig. S4. Pore size distribution of the three kerogen matrices at the initial state. (Pressure: 1 atm; Temperature: 298 $\mathrm{K})$ 
(a) 27 Macro-molecules

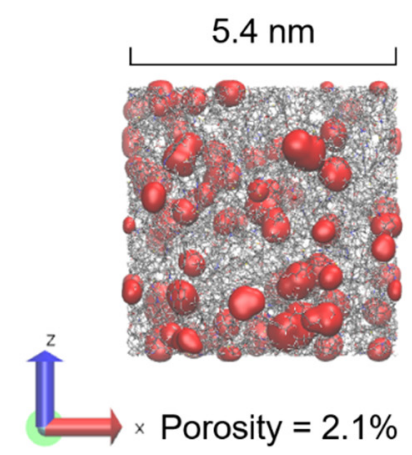

(b) 60 Macro-molecules

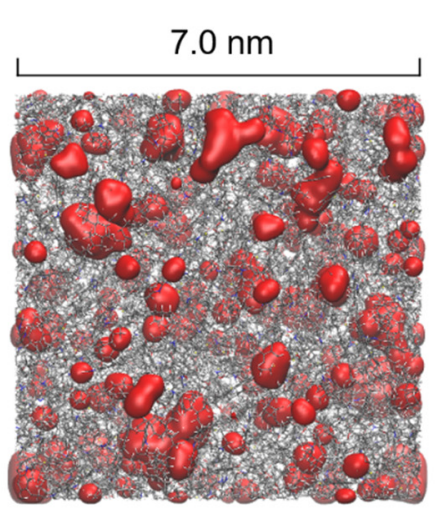

Porosity $=2.6 \%$ (c) 125 Macro-molecules

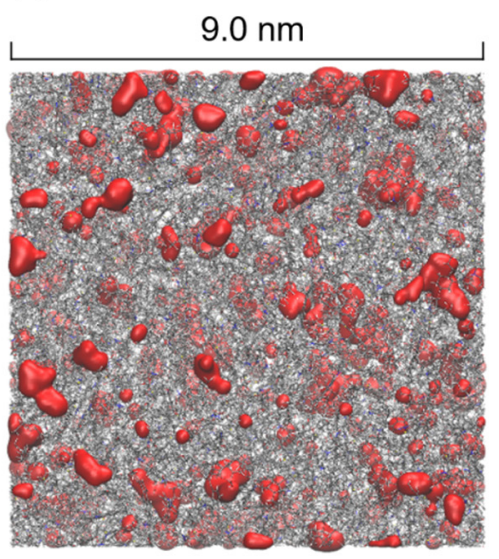

Porosity $=2.1 \%$

Fig. S5. Kerogen matrices (type II-A) with different domain sizes. The red color represents the pore space. Probe molecule: helium. 


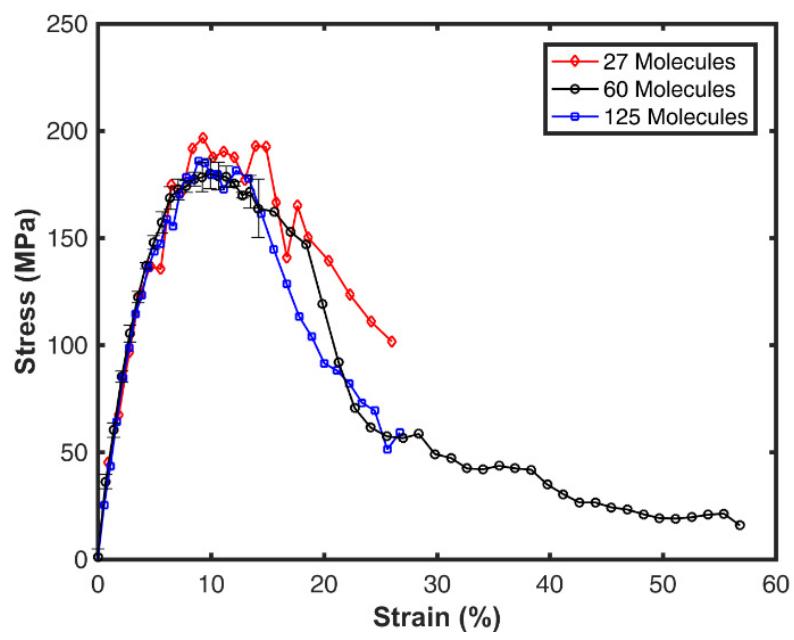

Fig. S6. Tensile stress-strain relationship of type II-A kerogen with three different domain sizes. 


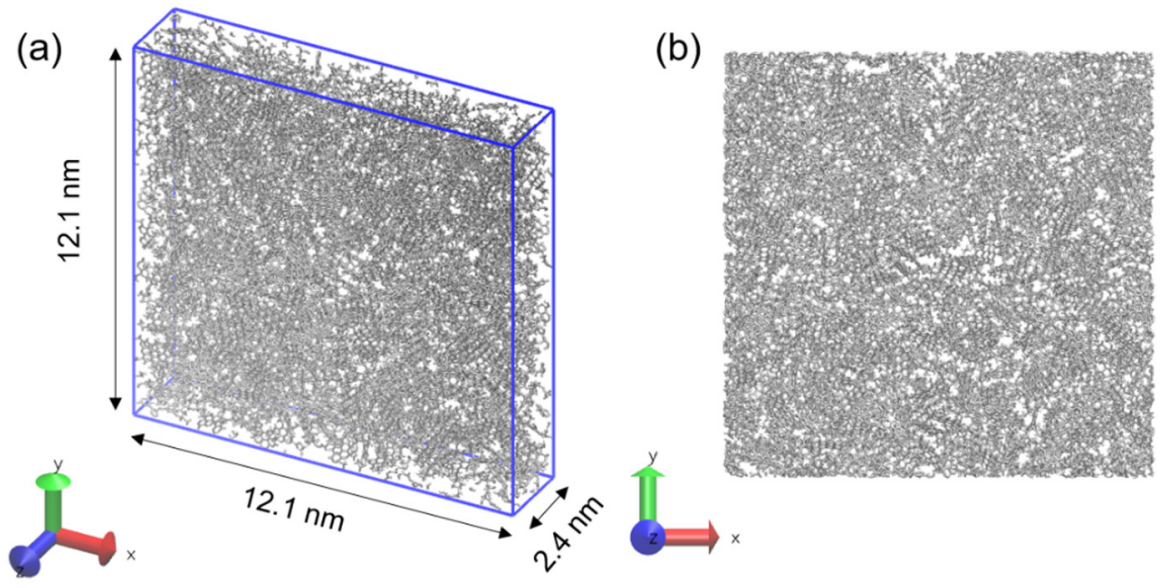

(c)

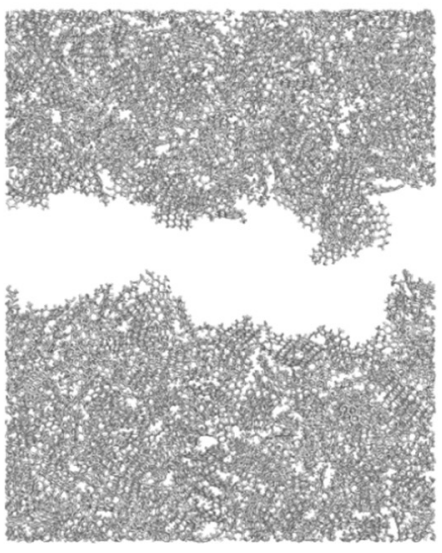

Fig. S7. Semi-2D kerogen matrix (type II-C) and the initial and final conditions of tensile failure. (a) Constructed semi-2D kerogen matrix with a larger size than in Fig. S2(e); (b) initial condition of the matrix; and (c) final condition after tensile failure. 


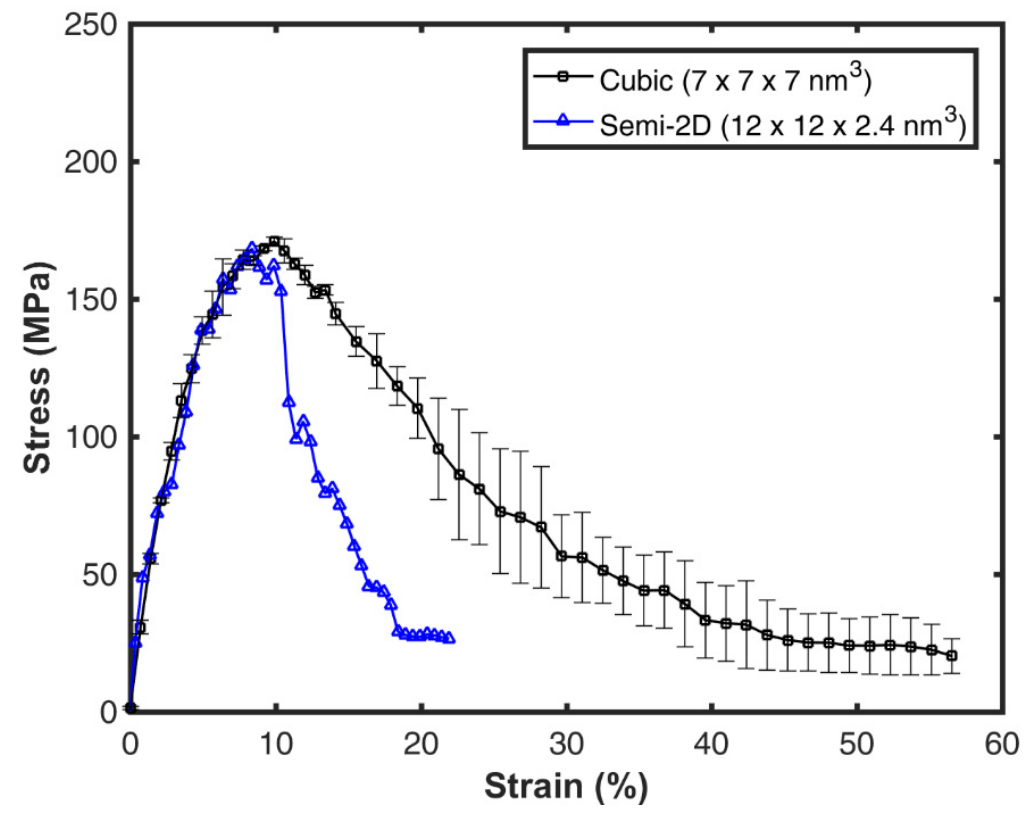

Fig. S8. Tensile stress-strain relationship of the type II-C kerogen matrices (cubic vs. semi-2D). 
(a) Type I-A

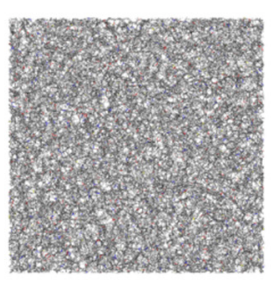

$\varepsilon=0 \%$

(b) Type II-A

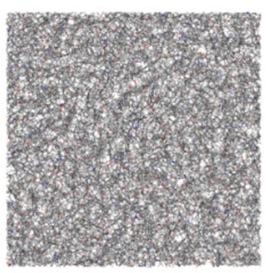

$\varepsilon=0 \%$

(c) Type III-A

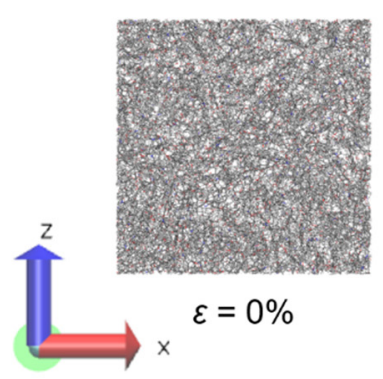

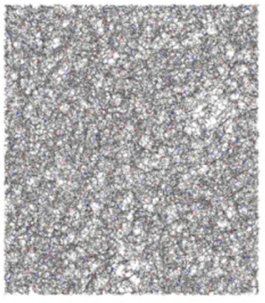

$\varepsilon=14 \%$

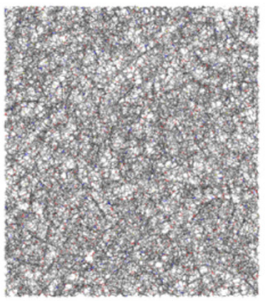

$\varepsilon=14 \%$

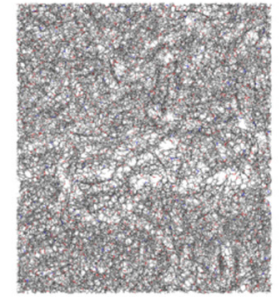

$\varepsilon=14 \%$

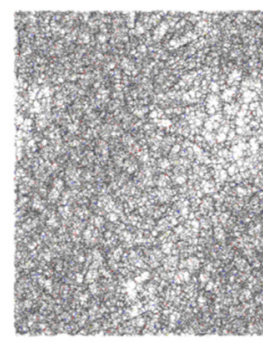

$\varepsilon=28 \%$

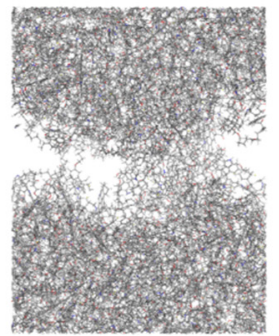

$\varepsilon=28 \%$

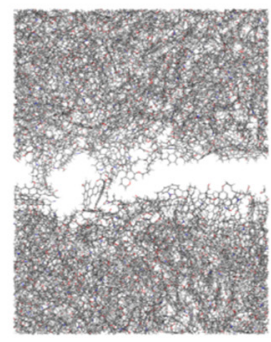

$\varepsilon=28 \%$

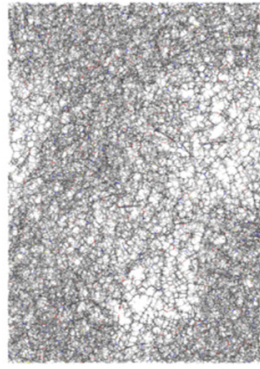

$\varepsilon=41 \%$

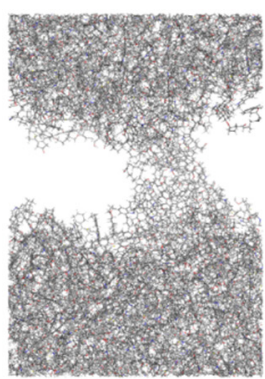

$\varepsilon=43 \%$
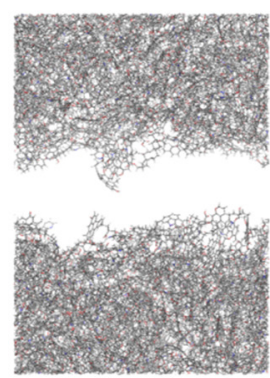

$\varepsilon=41 \%$

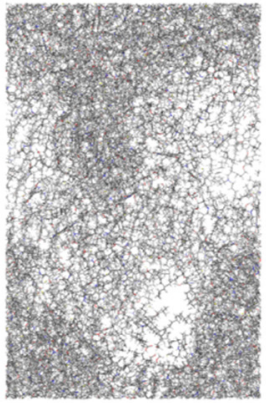

$\varepsilon=55 \%$
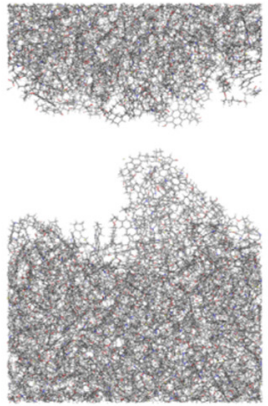

$\varepsilon=57 \%$
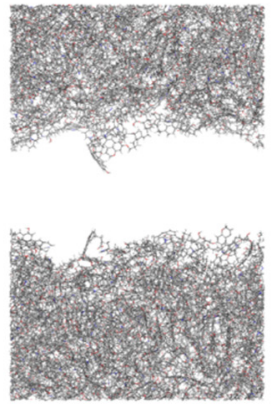

$\varepsilon=55 \%$

Fig. S9. Tensile failure of different kerogen types. Type I-A kerogen has fully ductile behavior. Type III-A kerogen shows an abrupt breakdown and relatively flat fracture surface, which reveals moderate brittle behavior. Type II-A kerogen has milder brittleness. 


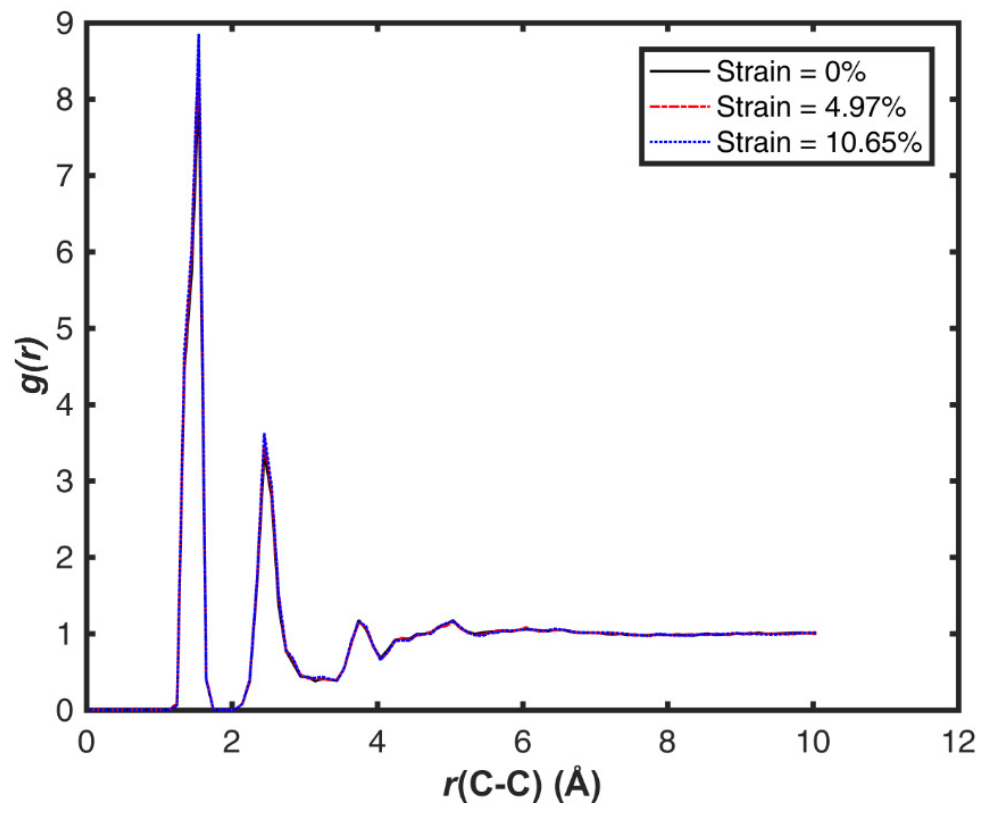

Fig. S10. Radial distribution function between carbon atoms. The yield point is around $\varepsilon=4.97 \%$, followed by a plastic region. The critical stress appears around $\varepsilon=10.65 \%$. 

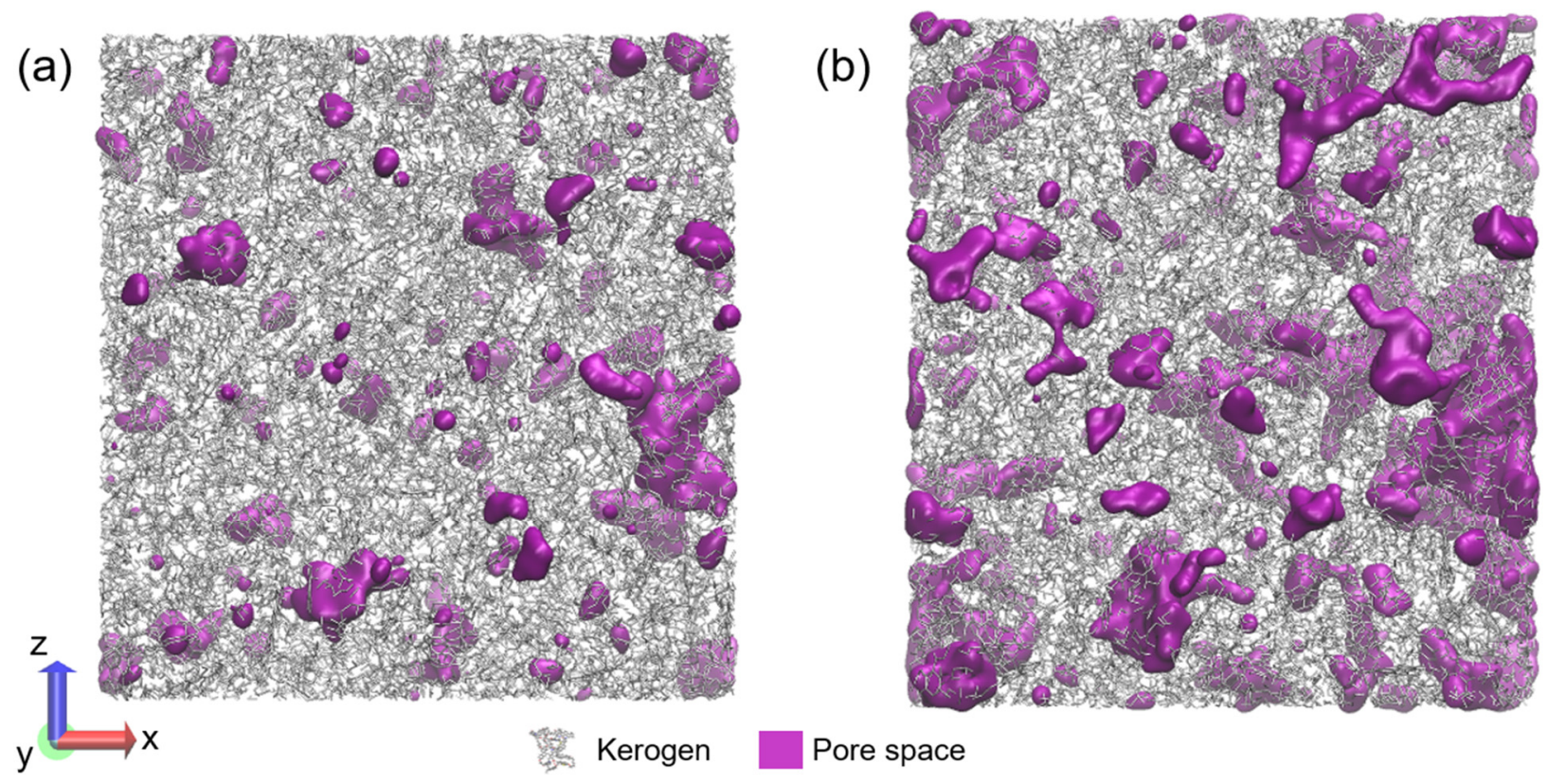

Fig. S11. Pore space evolution in the plastic region of type II-A kerogen matrix. (a) Strain $=4.97 \%$, porosity $=3.3 \%$, and surface area $=52.30 \mathrm{~m}^{2} / \mathrm{g}$; (b) strain $=10.65 \%$, porosity $=7.0 \%$, and surface area $=131.49 \mathrm{~m}^{2} / \mathrm{g}$. 


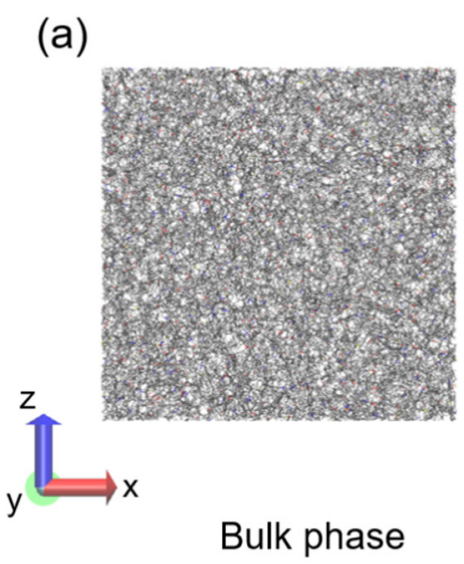

(b)

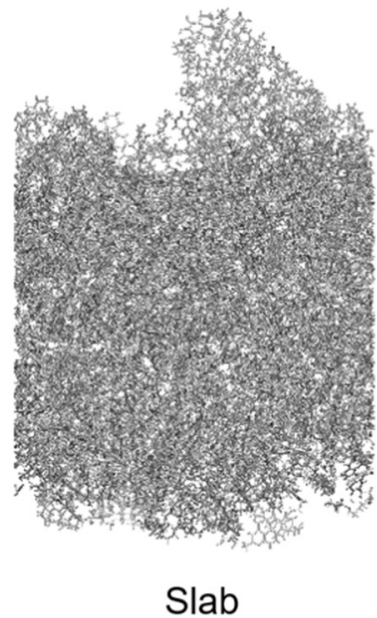

Fig. S12. Kerogen matrices (type II-A) for surface energy density calculation. (a) Periodic boundary conditions in all three directions; (b) periodic boundary condition in the $x$ - and $y$-directions. 


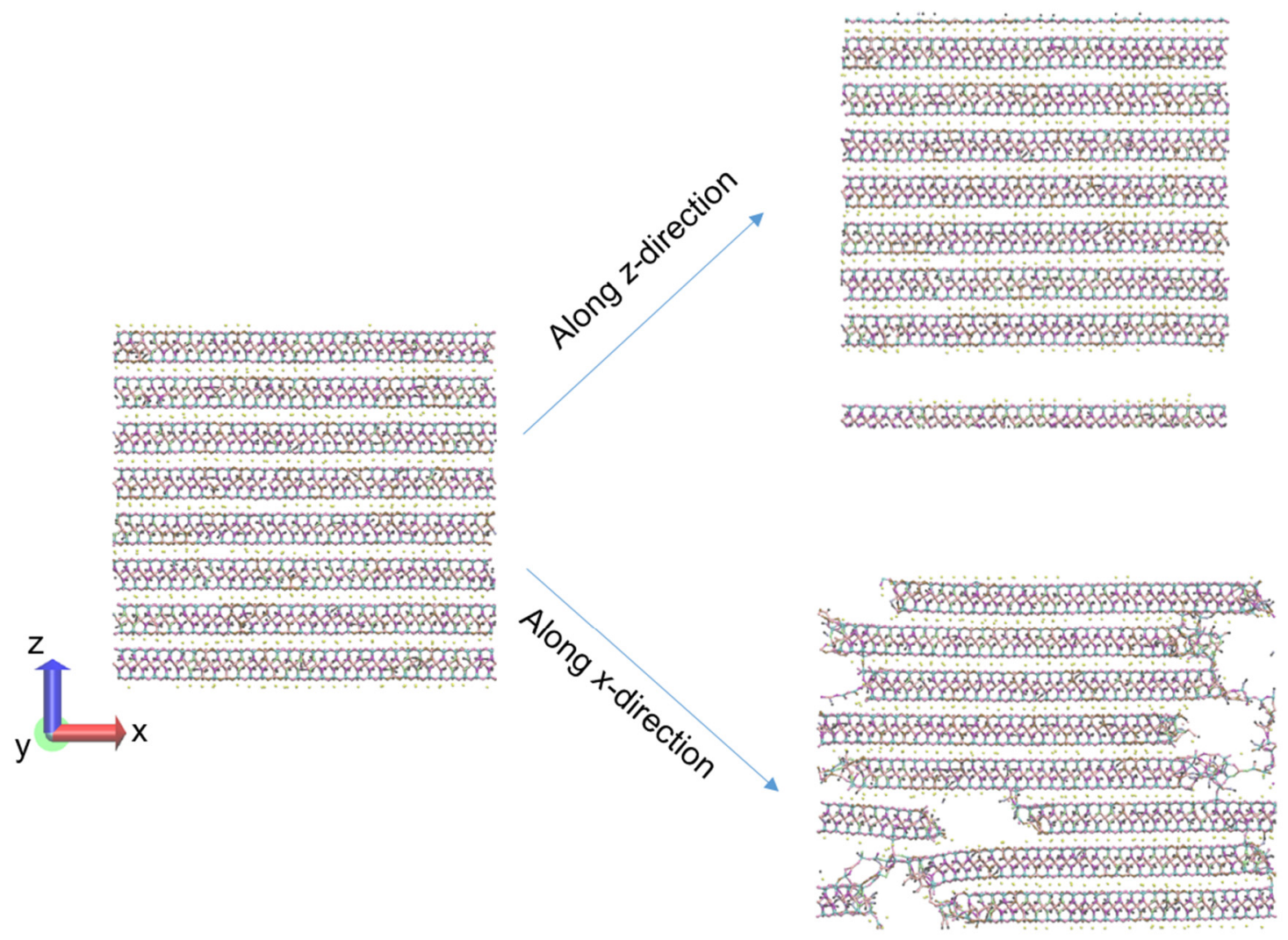

Fig. S13. Molecular structure of illite and the directions of tensile failure. The structure is created from the pyrophyllite unit cell $(16 \times 2 \times 8$ cells in the $x$-, $y$-, and $z$-directions $)$. The structure with random substitutions of $\mathrm{Al}(\mathrm{III})$ and $\mathrm{Si}(\mathrm{IV})$ are used. ${ }^{10}$ The extensions are performed in the directions perpendicular ( $z$-direction) and parallel ( $x$-direction) to the layers, respectively. 

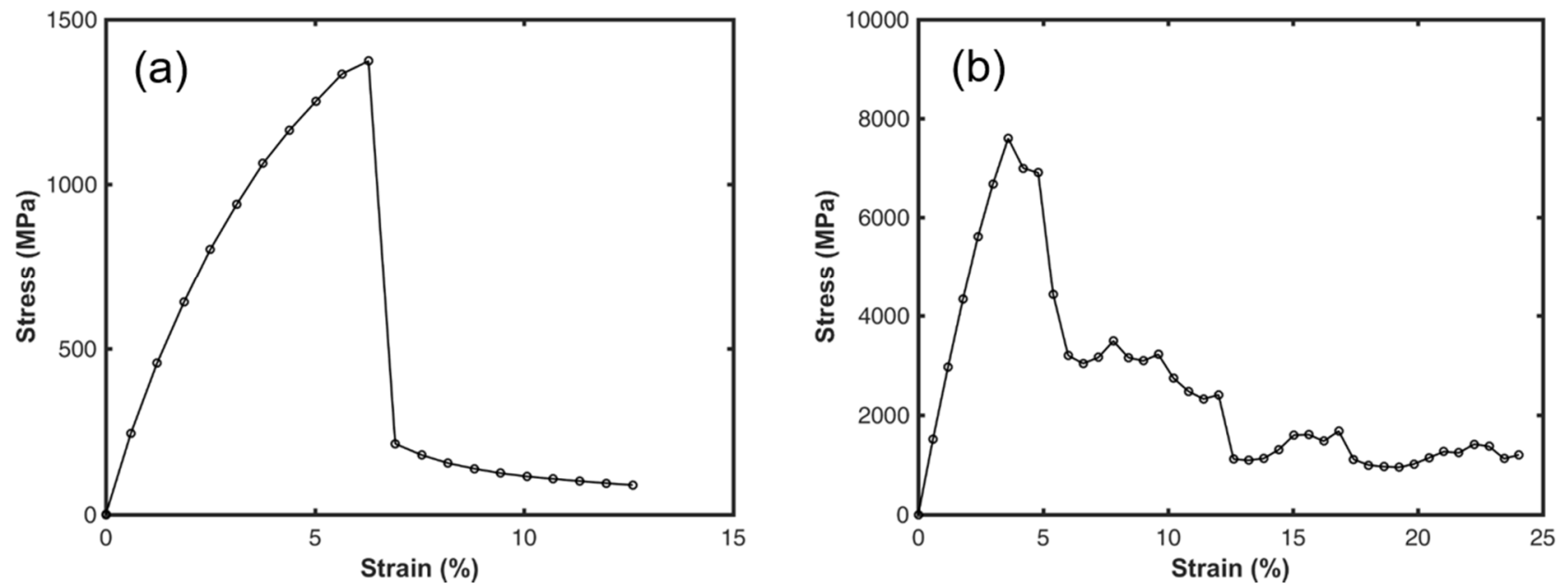

Fig. S14. Stress-strain relationships of illite. (a) Tensile failure in the direction perpendicular to the layers $(z-$ direction); (b) tensile failure in the direction parallel to the layers ( $x$-direction). 


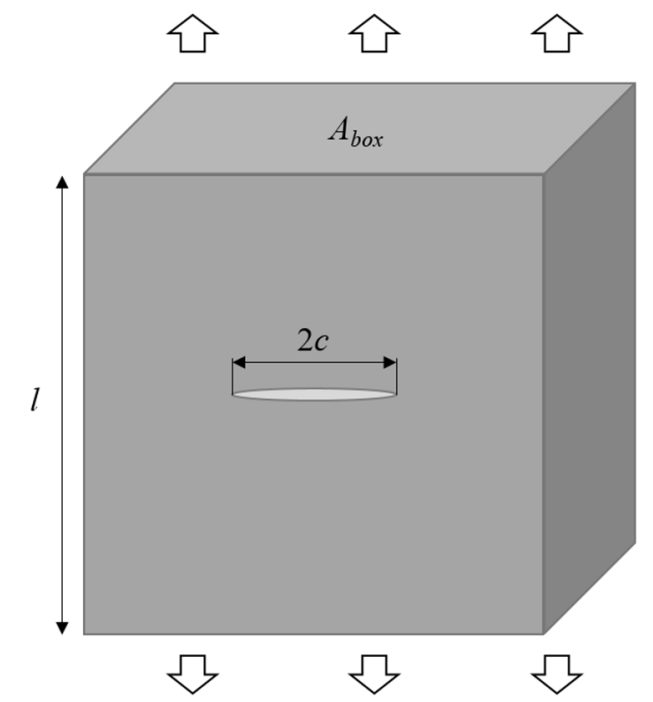

Fig. S15. Geometry of the sample with a crack length of $2 c$. 

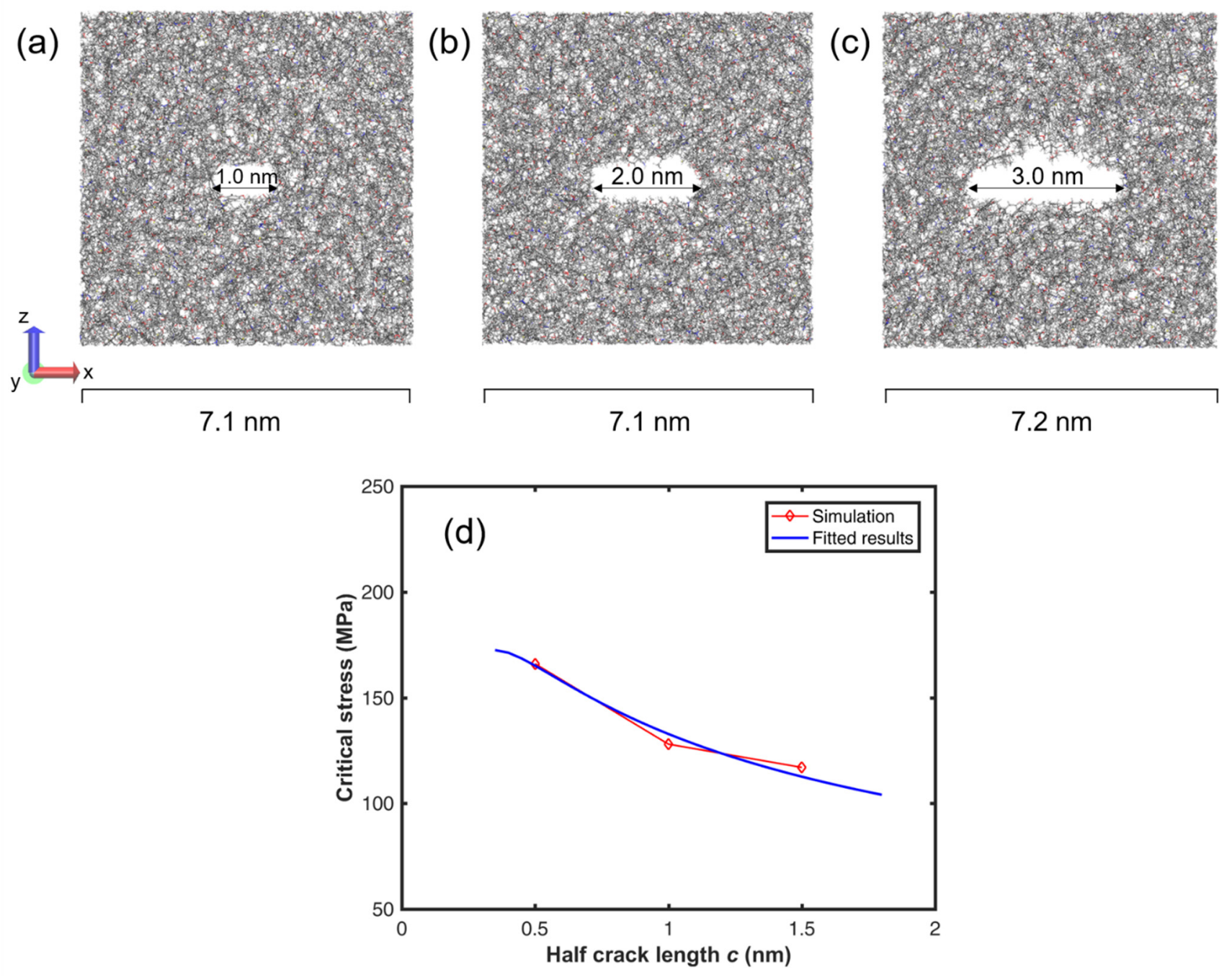

Fig. S16. Kerogen matrices containing pre-existing cracks with different lengths in the $x$-direction and the corresponding critical stresses. (a) - (c): The cracks have an aperture of $0.4 \mathrm{~nm}$ and the full length in the $y$-direction. (d) Critical stress from molecular simulations and fitting to Eqs. (S7) and (S8). 
Table S1. Annealing procedures for creating the kerogen matrix.

\begin{tabular}{cccc}
\hline Temperature $(\mathrm{K})$ & Pressure $($ bar $)$ & Time $(\mathrm{ps})$ & Time step $(\mathrm{fs})$ \\
\hline 300 to 1000 & 200 & 100 & 0.1 \\
1000 to 298.15 & 200 & 100 & 0.1 \\
298.15 & 0.1 & 100 & 0.1 \\
\hline
\end{tabular}




\section{References}

(1) Wu, T.; Firoozabadi, A. Effect of Microstructural Flexibility on Methane Flow in Kerogen Matrix by Molecular Dynamics Simulations. The Journal of Physical Chemistry C 2019, 123, 10874-10880.

(2) Herrera, L.; Do, D.; Nicholson, D. A Monte Carlo Integration Method to Determine Accessible Volume, Accessible Surface Area and Its Fractal Dimension. J. Colloid Interface Sci. 2010, 348, 529-536. (3) Wu, T.; Firoozabadi, A. Mechanical Properties and Failure Envelope of Kerogen Matrix by Molecular Dynamics Simulations. The Journal of Physical Chemistry C 2020, 124, 2289-2294.

(4) Ungerer, P.; Collell, J.; Yiannourakou, M. Molecular Modeling of the Volumetric and Thermodynamic Properties of Kerogen: Influence of Organic Type and Maturity. Energy Fuels 2015, 29, 91-105.

(5) Irwin, G. R. Plastic Zone near a Crack and Fracture Toughness. In Proceedings of the Seventh Sagamore Ordnance Materials Research Conference: Mechanical and Metallurgical Behavior of Sheet Materials, 1960; pp 63-78.

(6) Anderson, T. L. Fracture Mechanics: Fundamentals and Applications; CRC Press: Boca Raton, 2005.

(7) Zhao, J.; Zhang, D.; Wu, T.; Tang, H.; Xuan, Q.; Jiang, Z.; Dai, C. Multiscale Approach for Mechanical Characterization of Organic-Rich Shale and Its Application. Int. J. Geomech. 2019, 19, 04018180.

(8) Bousige, C., et al. Realistic Molecular Model of Kerogen's Nanostructure. Nature Materials 2016, $15,576-582$.

(9) Song, D.-P.; Liang, Y.-C.; Chen, M.-J.; Bai, Q.-S. Molecular Dynamics Study on Surface Structure and Surface Energy of Rutile TiO2 (1 110$)$. Appl. Surf. Sci. 2009, 255, 5702-5708.

(10) Hantal, G.; Brochard, L.; Laubie, H.; Ebrahimi, D.; Pellenq, R. J.-M.; Ulm, F.-J.; Coasne, B. Atomic-Scale Modelling of Elastic and Failure Properties of Clays. Mol. Phys. 2014, 112, 1294-1305.

(11) Meunier, A.; Velde, B.; Velde, B. Illite: Origins, Evolution and Metamorphism; Springer Science \& Business Media, 2004.

(12) Griffith, A. A. The Theory of Rupture. In Proceedings of the First International Congress for Applied Mechanics, 1924; pp 55-63.

(13) Irwin, G. R. Fracture. In Elasticity and Plasticity / Elastizität Und Plastizität, Flügge, S., Ed. Springer: Berlin, Heidelberg, 1958; pp 551-590.

(14) Fjar, E.; Holt, R. M.; Raaen, A.; Risnes, R.; Horsrud, P. Petroleum Related Rock Mechanics; Elsevier, 2008; Vol. 53. 